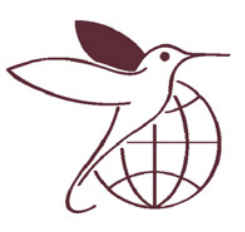

International Diabetes Federation

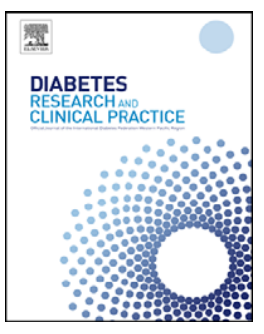

\title{
Sports activity and risk of type 2 diabetes in Chinese
}

\author{
Kuo-Liong Chien ${ }^{a, b}$, Ming-Fong Chen ${ }^{b}$, Hsiu-Ching Hsu ${ }^{b}$, Ta-Chen Su ${ }^{b}$, Yuan-Teh Lee, ${ }^{b, *}$ \\ a Institute of Preventive Medicine, National Taiwan University, Taipei, Taiwan \\ ${ }^{\mathrm{b}}$ Department of Internal Medicine, National Taiwan University Hospital, Taipei, Taiwan
}

\section{A R T I C L E I N F O}

Article history:

Received 8 September 2008

Received in revised form

3 March 2009

Accepted 6 March 2009

Keywords:

Physical activity

Type 2 diabetes

Community

Chinese

\begin{abstract}
A B S T R A C T
An association between physical inactivity and obesity risk has been established. However, the relationship between sports activity and the risk of diabetes among Chinese populations is still unclear. We prospectively investigated the association between sports activity and incidence of type 2 diabetes in a Chinese population. Habitual physical activity in the presumed last year was obtained from a questionnaire developed by Baecke and colleagues. During a median 9.02-year follow-up period among the 1936 participants with complete questionnaire data, 312 participants developed diabetes. After adjusting for age, gender and body mass index, the relative risk (RR) of diabetes according to quartiles of sports activity were 0.86, 0.71 and 0.72 (95\% Confidence Interval [CI], 0.52-0.99, P for trend, 0.048). Participants with the highest quartiles of sports activity had a significantly lower risk than the participants with the lowest quartiles among the obese group (multivariate RR, $0.61,95 \% \mathrm{CI}$, $0.41-0.92$, $P$ for trend $=0.018$ ). Furthermore, the RRs of diabetes were 2.24 (95\% CI, 1.58-3.19) for participants who were obese and sedentary, compared with those who were of a healthy weight and participated actively in sports. The findings suggest a significant inverse association between sports activity and incidence of type 2 diabetes in middle to olderaged Chinese individuals.
\end{abstract}

(C) 2009 Published by Elsevier Ireland Ltd.

\section{Introduction}

Type 2 diabetes epidemics are a major public health concern, causing tens of millions of chronic illnesses and a significant number of deaths worldwide each year. The prevalence of type 2 diabetes has increased substantially around the world [1] and primary prevention through lifestyle intervention, such as weight reduction and increased physical activity, is considered important for prevention and control of type 2 diabetes [2]. The association of habitual physical activity and the risk of type 2 diabetes has been reported in Western [3-5] and Asian countries [6,7]. In addition, low physical activity has been associated with all-cause mortality [8], cardiovascular mortality [9-12], stroke [13,14] and biomarker profiles $[15,16]$.
Therefore, understanding the relationship between physical activity and type 2 diabetes is an important way to facilitate primary prevention of type 2 diabetes.

Physical activity probably improves diabetes prevention through multiple pathways including body weight control, increasing energy expenditure and reducing fat mass [17]. The categories of habitual physical activity include occupational, transportation, household, sports and leisure-time activities [18] and the type and intensity of physical activity varies in the different categories. Many studies to date have focused on sedentary and leisure-time activities and the risk of type 2 diabetes $[3,19,20]$. Based on 70658 women who were free of diabetes and followed up for 4.6 years, Villegas et al. demonstrated that leisure-time and daily physical activities

${ }^{*}$ Corresponding author at: Department of Internal Medicine, National Taiwan University Hospital, Taipei 100, Taiwan. Tel.: +886 23366 8017; fax: +886 223920456.

E-mail address: ytlee@ntu.edu.tw (Y.-T. Lee).

0168-8227/\$ - see front matter (C) 2009 Published by Elsevier Ireland Ltd. doi:10.1016/j.diabres.2009.03.006 
are inversely associated with type 2 diabetes risk [7]. Another study based on 2924 Japanese male workers also showed a similar association between daily physical activity and diabetes risk [6]. However, the role of sports activity in diabetes risk among Chinese populations is unclear. In this study we investigate the roles of sports, leisure-time and work physical activity on the risk of diabetes among middle-aged and elderly adults from a community-based cohort.

\section{Subjects, materials and methods}

\subsection{Study design and participants}

Details of this cohort study have been published previously [21-23]. Briefly, the Chin-Shan community cardiovascular cohort study (CCCC) began in 1990 by recruiting 1703 men and 1899 women of Chinese ethnicity aged 35 years old and above from the Chin-Shan township, $30 \mathrm{~km}$ north of metropolitan Taipei, Taiwan. Information about anthropometry, lifestyle, and medical conditions was assessed by interview questionnaires in 2-year cycles for the initial 6 years and the validity and reproducibility of the collected data and measurements have been reported in detail elsewhere [24]. Participants with baseline incomplete blood data $(n=41)$, diagnosis of diabetes (fasting glucose $\geq 126 \mathrm{mg} / \mathrm{dL}$ or with hypoglycemic medication history, $n=473)$, and cardiovascular disease and cancer history $(n=170)$ were excluded from this investigation. Incident diabetes cases were defined by fasting glucose levels $\geq 126 \mathrm{mg} / \mathrm{dL}$ or by the use of oral hypoglycemic or insulin medication [25] and The National Taiwan University Hospital Committee Review Board approved the study protocol.

\subsection{Physical activity levels measurement}

We used the Baecke questionnaire of habitual physical activity, which has been validated previously and shown to be an easily administered instrument [26,27]. This questionnaire was originally developed in a Dutch population, has good reliability and moderate validity [27], and has been implemented in an elderly Taiwanese population [28]. The questionnaire consists of sports (exercise), leisure and work activities, and is scored on a 5-point Likert scale, ranging from never to sometimes or very often. Additional questions report the type of sports activity and both the number of hours per week and the number of months per year in which the participants were involved in that activity. The scoring of the questionnaire included specific scoring criteria for each of the three sections: sports, leisure and work activities. We calculated the amount of physical activity according to Baecke's proposal [26].

We limited the study participants to the 1639 individuals who completed the physical activity questionnaire in 1992. Compared with non-participants $(n=1321)$, the participants were more likely to be female ( $56 \%$ vs. $44 \%$ ) and older ( 54.7 vs. 53.2 years old), and had a higher rate of hypertension (29\% vs. 24\%). The distributions of body mass index and HDLcholesterol were similar between participants and nonparticipants $(P=0.27$ for body mass index and 0.22 for HDLcholesterol).

\subsection{Measurement of biochemical markers}

The procedure for blood collection has been reported elsewhere $[29,30]$. Briefly, all venous blood samples drawn after a 12-hour overnight fast were immediately refrigerated and transported within $6 \mathrm{~h}$ to the National Taiwan University Hospital. Serum samples were then stored at $-70^{\circ} \mathrm{C}$ before batch assay for levels of total cholesterol, triglycerides, and high density lipoprotein cholesterol (HDL-C). Standard enzymatic tests for serum cholesterol and triglycerides were used (Merck 14354 and 14366, Germany, respectively). HDL-C levels were measured in supernatants after the precipitation of specimens with magnesium chloride phosphotungstate reagents (Merck 14993). LDL-C concentrations were calculated as total cholesterol minus cholesterol in the supernatant by precipitation method (Merck 14992)[31]. Blood samples for glucose analysis were extracted into glass test tubes each containing $80 \mathrm{~mol} / \mathrm{L}$ fluoride/oxalate reagents. After centrifugation at $4{ }^{\circ} \mathrm{C}, 1500 \times \mathrm{g}$ for $10 \mathrm{~min}$, glucose levels were measured in the supernatant by enzymatic assay (Merck 3389 commercial kit, Germany) in a Eppendorf 5060 autoanalyzer. We followed the criteria of the adult treatment panel III guidelines to define metabolic syndrome [32], using Asianspecific waist circumference cutpoints [33].

\subsection{Statistical analysis}

Participants were categorized on the basis of quartiles of sports activity, and continuous variables are presented as mean values; categorical data are presented in a contingency table with ANOVA to test the difference among quartiles. Relationships between various baseline physical activities and body mass index as well as lipids were examined by age and gender adjusted Spearman's partial correlation coefficients.

Incidence rates of diabetes were calculated by dividing cases by the person-years of follow-up for each quartile of physical activity. The relative risk (RR) of diabetes was calculated by dividing the incidence rate of each quartile by the rate in the first quartile. We used Cox proportionalhazards models to adjust for potential confounding variables. We specified four models to estimate the relative risk of diabetes in the higher physical activity quartiles compared with the lowest quartile. In multivariate analyses, we first adjusted for age groups (35-44, 45-54, 55-64, 65-74, $\geq 75$ years old) and gender (Model 1). We then additionally adjusted for body mass index $\left(<18,18-20.9,21-22.9,23-24.9\right.$, or $\left.\geq 25 \mathrm{~kg} / \mathrm{m}^{2}\right)$ for Model 2. We included the metabolic syndrome, as an additional variable in Model 2, for Model 3. Finally, we added lifestyle factors, including alcohol intake (non-drinker/current), smoking (yes/no), marital status (single, married, or divorced), educational level ( $<9$ years, $\geq 9$ years), occupation (no work, manual work, or professional), and family history of diabetes (yes/no), hypertension, HDL-C, triglycerides and fasting glucose levels in Model 4 as potential confounding factors. In all analyses, we modeled the sports, leisure, and work activity variables in quartiles to avoid a linearity assumption and to reduce the effect of outliers. To test for linear trend across physical activity quartiles, we used the median values for the categories and fit the continuous variable in the model. We also tested the goodness of fit for the 
model by using the Hosmer and Lemeshow test [34]. In addition, we conducted analyses of sports activity and diabetes risk stratified by gender, age and body mass index, according to their median values. Likelihood ratio tests were conducted to test statistical interactions between sports activity and gender, age and body mass index by comparing the $-2 \log$ (likelihood) between two nested models, one with only main effects and the other with both the main effects and interaction terms.

All statistical tests were two-tailed with a type I error of 0.05 , and $P$ values $<0.05$ were considered statistically significant. Analyses were performed with SAS version 9.1 (SAS Institute, Cary, NC) and Stata version 9.1 (Stata Corporation, College Station, Texas).

\section{Results}

Participants in the highest sports activity quartile were more likely to be male, current alcohol drinkers, have a higher rate of hypertension, and be unemployed, compared with those in the lower quartiles (Table 1). Participants in the third quartile of sports activity had the highest prevalence of education level $(\geq 9$ years, $8.5 \%, P=0.036$ ). Similarly, participants in the highest quartile were more likely to have a higher age, work and leisure activities, yet lower HDL-cholesterol levels. The distribution of smokers, diabetic family history, metabolic syndrome, body mass index, and glucose levels were similar across the quartiles.

We documented 312 incident cases of diabetes during a median 9.02-year (inter-quartile range: 4.99-9.94 year) followup in the 1639 subjects. Table 2 shows the relative risks and $95 \%$ confidence intervals (CI) of diabetes according to quartiles of sports activity at baseline. After adjusting for age, gender and body mass index, the RRs of diabetes in the higher quartiles of sports activity were 0.86 (95\% CI, 0.64-1.16), 0.71 (95\% CI, 0.53-0.96) and 0.72 (95\% CI, 0.52-0.99, P for trend, $0.048)$, compared with the lowest quartile. Additional adjustments for metabolic syndrome, smoking, current alcohol drinking, socioeconomic status, hypertension status, HDL-C, triglycerides, fasting glucose levels and family history of diabetes did not change appreciably the magnitude of risks: the RR of diabetes for the higher sports activity quartiles were 0.82 (95\% CI, 0.60-1.12), 0.65 (95\% CI, 0.47-0.89) and 0.68 (95\% CI, $0.49-0.95, P$ for trend $=0.028)$. However, the risks of diabetes according to work activity and leisure activity did not reach significant levels. Our findings suggested that only sports activity, but not work or leisure activity, was associated with a decreased risk of diabetes.

To address the possibility that diabetes risk may have varied according to gender, age and obesity status, we conducted

Table 1 - Characteristics of the population at baseline (1990) according to quartiles of sports activity among the 1639 participants with available questionnaire data.

\begin{tabular}{|c|c|c|c|c|c|}
\hline & \multicolumn{4}{|c|}{ Quartiles of sports activity } & \multirow[t]{3}{*}{$P$} \\
\hline & 1 & 2 & 3 & 4 & \\
\hline & $n=454(\%)$ & $n=390(\%)$ & $n=446(\%)$ & $n=349(\%)$ & \\
\hline Gender & & & & & 0.006 \\
\hline Women & 60.8 & 59.2 & 53.8 & 49.6 & \\
\hline Men & 39.2 & 40.8 & 46.2 & 50.4 & \\
\hline Current smoker (yes) & 28.6 & 30.3 & 34.1 & 36.4 & 0.08 \\
\hline Alcohol drinking (yes) & 24.0 & 23.3 & 29.4 & 30.7 & 0.039 \\
\hline Marital status & & & & & 0.011 \\
\hline Single & 2.9 & 0.8 & 2.7 & 2.3 & \\
\hline Live with spouse & 89.6 & 88.6 & 87.2 & 82.8 & \\
\hline Divorced or separated & 7.5 & 10.6 & 10.1 & 14.9 & \\
\hline Education level & & & & & 0.036 \\
\hline$<9$ years & 95.6 & 95.4 & 91.5 & 94.3 & \\
\hline$\geq 9$ years & 4.4 & 4.6 & 8.5 & 5.7 & \\
\hline Job status & & & & & 0.007 \\
\hline No job & 50.0 & 46.9 & 48.9 & 59.6 & \\
\hline Farmer, labor work & 33.3 & 36.9 & 31.4 & 25.5 & \\
\hline Professional, business & 16.7 & 16.2 & 19.7 & 14.9 & \\
\hline Family history of diabetes & 7.7 & 11.8 & 9.2 & 6.9 & 0.08 \\
\hline Hypertension & 29.5 & 20.6 & 26.7 & 39.7 & $<0.0001$ \\
\hline Metabolic syndrome & 18.2 & 16.0 & 18.4 & 22.2 & 0.19 \\
\hline Age & 53.5 & 52.7 & 54.4 & 58.8 & $<0.0001$ \\
\hline Body mass index & 23.4 & 23.3 & 23.2 & 23.5 & 0.44 \\
\hline HDL-cholesterol & 48.7 & 49.3 & 48.9 & 46.8 & 0.042 \\
\hline Glucose & 101.5 & 102.4 & 102.1 & 101.9 & 0.56 \\
\hline Sports activity & 1.62 & 2.00 & 2.35 & 3.17 & $<0.0001$ \\
\hline Leisure activity & 2.39 & 2.40 & 2.61 & 2.80 & $<0.0001$ \\
\hline Work activity & 2.72 & 2.82 & 2.82 & 2.83 & $<0.0001$ \\
\hline Overall physical activity & 6.73 & 7.22 & 7.78 & 8.79 & $<0.0001$ \\
\hline
\end{tabular}


Table 2 - Median levels, numbers, incident cases, person-years, rates, and relative risks with $95 \%$ confidence intervals for various physical activity quartiles for the association with type 2 diabetes in the study participants, 1990-2000.

Q1

Q2

Q3

Q4

$P$, trend

Sports activity

Median

Cases

Person-year

Rate/1000

Model 1

Model 2

Model 3

Model 4

Work activity

Median

Cases

Person-year

Rate/1000

Model 1

Model 2

Model 3

Model 4

Leisure activity

Median

Cases

Person-year

Rate/1000

Model 1

Model 2

Model 3

Model 4

$\begin{array}{ll}1.75 & 2 \\ 100 & 76 \\ 3430.6 & 3038.4 \\ 29.1 & 25 \\ 1 & 0.83(0.62-1.12) \\ 1 & 0.86(0.64-1.16) \\ 1 & 0.85(0.63-1.15) \\ 1 & 0.82(0.60-1.12)\end{array}$

2.375

62

2048.5

30.3

1

1

1

1

2

81

3538.5

22.9

1

1

1

1
2.75

84

3967.3

21.2

$0.70(0.51-0.98)$

$0.74(0.53-1.03)$

$0.73(0.53-1.02)$

$0.75(0.54-1.05)$

\subsection{5}

88

3415.4

25.8

$1.11(0.82-1.50)$

$1.11(0.82-1.50)$

$1.05(0.78-1.43)$

$1.09(0.80-1.49)$
$2.25 \quad 3$

$75 \quad 61$

$3498.6 \quad 2598.8$

$21.4 \quad 23.5$

$0.70(0.52-0.94) \quad 0.74(0.54-1.03) \quad 0.084$

$0.71(0.53-0.96) \quad 0.72(0.52-0.99) \quad 0.048$

$0.67(0.49-0.90) \quad 0.68(0.49-0.95) \quad 0.023$

$\begin{array}{lll}0.65(0.47-0.89) & 0.68(0.49-0.95) & 0.028\end{array}$

$2.875 \quad 3.125$

$48 \quad 94$

$2267.1 \quad 3407.3$

$21.2 \quad 27.6$

$\begin{array}{lll}0.72(0.49-1.05) & 0.88(0.64-1.22) & 0.58\end{array}$

$0.75(0.51-1.10) \quad 1.00(0.72-1.39) \quad 0.87$

$0.73(0.49-1.07) \quad 0.96(0.69-1.33) \quad 0.90$

$0.75(0.50-1.12) \quad 0.97(0.69-1.37) \quad 0.98$

$2.75 \quad 3.5$

$79 \quad 57$

$3121.8 \quad 2175.7$

$25.3 \quad 26.2$

$1.11(0.81-1.51) \quad 1.10(0.78-1.55) \quad 0.65$

$1.13(0.83-1.55) \quad 1.13(0.81-1.60) \quad 0.51$

$1.09(0.80-1.49) \quad 1.18(0.84-1.66) \quad 0.35$

$\begin{array}{lll}1.07(0.77-1.48) & 1.24(0.87-1.75) & 0.29\end{array}$

Model 1: adjusted for age groups $(35-44,45-54,55-64,65-74, \geq 75$ years old) and gender.

Model 2: Model 1 plus body mass index $\left(<18,18-20.9,21-22.9,23-24.9\right.$, or $\left.\geq 25 \mathrm{~kg} / \mathrm{m}^{2}\right)$.

Model 3: Model 2 plus metabolic syndrome.

Model 4: Model 3 plus smoking, current alcohol drinking (regular/no), marital status (single, married and living with spouse, or divorced and separated), education level (less than 9 years, at least 9 years), occupation (no work, labor, official or business), hypertension status, HDL-C, triglycerides, glucose levels and family history of diabetes.

additional stratified analyses (Table 3). We found that the participants in the highest quartiles of sports activity had a significantly lower risk than the participants in the lowest quartiles, which was primarily attributable to the presence of obesity (multivariate RR, $0.61,95 \% \mathrm{CI}<0.41-0.92$, $P$ for trend $=0.018$ ). However, we did not find any significant association in the absence of obesity. The relationship between sports activity and diabetes was not modified as a result of gender, age or body mass index level (all $P$ values $>0.2$ for all comparisons).

In a joint analysis of body mass index and sports activity, taking participants who had a healthy weight and were in the active sports activity as the reference group, the multivariate RRs of diabetes were 2.24 (95\% CI, 1.58-3.19) for participants who were obese and sedentary, 1.41 (95\% CI, 1.03-1.92) for those who were active but obese, and 1.08 (95\% CI, 0.68-1.70) for those who had a healthy weight but were sedentary (Fig. 1).

\section{Discussion}

Our data suggested an inverse association between sports activity and incidence of type 2 diabetes in middle aged to elderly Chinese.
Among postmenopausal women in the Women's Health Initiative Study who were followed for 8 years, an inverse association between sports activity and self-reported diabetes was seen, the effects most apparent among Caucasian women [35]. Among 4369 middle-aged Finnish adults who were followed for 9.4 years, an inverse association between physical activity and diabetes was seen, the association persistent in individuals who were obese and had elevated glucose levels [36]. Another 12-year cohort study on 6898 Finnish men and women also demonstrated that moderate and high occupational, commuting or leisure-time physical activity was inversely associated with diabetes risk [3]. The jointed additive effects of physical activity and body mass index were apparent in these Finnish adults [37]. However, in another cohort with 15.5 years of follow-up data among Canadian adults, leisuretime physical activity did not predict incident diabetes [38], although sports-related activities were not measured. The most detailed prospective analysis of various physical activities and diabetes is that conducted by $\mathrm{Hu}$ et al. using 6-years of follow-up data from the Nurses' Health Study [39]. In that study, sedentary behavior, especially watching television, was associated with an elevated risk of obesity and type 2 diabetes. Furthermore, more than $90 \%$ of diabetes cases have been attributed to habits and behavior which do not conform 
Table 3 - Relative risks with 95\% confidence intervals for various physical activity quartiles for the association with type 2 diabetes in the study participants, stratified by different gender, age groups and body mass index levels, 1990-2000.

$$
\text { Q1 Q2 Q3 }
$$

Q4 $P$, trend

\begin{tabular}{|c|c|c|c|c|c|c|c|c|c|c|c|}
\hline \multicolumn{12}{|l|}{ Gender } \\
\hline \multicolumn{12}{|l|}{ Women } \\
\hline Model 1 & 1 & 0.86 & 0.58 & 1.28 & 0.66 & 0.43 & 1.01 & 0.70 & 0.44 & 1.12 & 0.11 \\
\hline Model 2 & 1 & 0.83 & 0.56 & 1.24 & 0.65 & 0.43 & 1.00 & 0.73 & 0.46 & 1.17 & 0.17 \\
\hline Model 3 & 1 & 0.80 & 0.54 & 1.20 & 0.61 & 0.40 & 0.94 & 0.72 & 0.45 & 1.14 & 0.14 \\
\hline Model 4 & 1 & 0.78 & 0.52 & 1.18 & 0.61 & 0.39 & 0.94 & 0.66 & 0.41 & 1.08 & 0.09 \\
\hline \multicolumn{12}{|l|}{ Men } \\
\hline Model 1 & 1 & 0.77 & 0.49 & 1.22 & 0.74 & 0.48 & 1.14 & 0.80 & 0.51 & 1.26 & 0.48 \\
\hline Model 2 & 1 & 0.84 & 0.53 & 1.34 & 0.79 & 0.51 & 1.23 & 0.74 & 0.47 & 1.18 & 0.26 \\
\hline Model 3 & 1 & 0.87 & 0.55 & 1.38 & 0.73 & 0.47 & 1.13 & 0.70 & 0.44 & 1.12 & 0.16 \\
\hline Model 4 & 1 & 0.79 & 0.49 & 1.28 & 0.67 & 0.43 & 1.06 & 0.73 & 0.46 & 1.18 & 0.26 \\
\hline \multicolumn{12}{|l|}{ Age group } \\
\hline \multicolumn{12}{|l|}{ Age $<53$} \\
\hline Model 1 & 1 & 0.95 & 0.63 & 1.42 & 0.73 & 0.47 & 1.12 & 0.73 & 0.42 & 1.27 & 0.17 \\
\hline Model 2 & 1 & 1.00 & 0.67 & 1.49 & 0.76 & 0.49 & 1.17 & 0.73 & 0.42 & 1.27 & 0.17 \\
\hline Model 3 & 1 & 1.02 & 0.68 & 1.53 & 0.71 & 0.46 & 1.11 & 0.77 & 0.44 & 1.34 & 0.20 \\
\hline Model 4 & 1 & 0.92 & 0.60 & 1.41 & 0.65 & 0.41 & 1.03 & 0.86 & 0.48 & 1.54 & 0.35 \\
\hline \multicolumn{12}{|l|}{ Age $\geq 53$} \\
\hline Model 1 & 1 & 0.73 & 0.46 & 1.14 & 0.71 & 0.47 & 1.08 & 0.77 & 0.51 & 1.16 & 0.41 \\
\hline Model 2 & 1 & 0.70 & 0.45 & 1.10 & 0.69 & 0.45 & 1.04 & 0.72 & 0.48 & 1.09 & 0.25 \\
\hline Model 3 & 1 & 0.68 & 0.44 & 1.07 & 0.64 & 0.42 & 0.97 & 0.65 & 0.43 & 0.99 & 0.12 \\
\hline Model 4 & 1 & 0.70 & 0.44 & 1.11 & 0.63 & 0.41 & 0.98 & 0.63 & 0.41 & 0.97 & 0.09 \\
\hline \multicolumn{12}{|l|}{ BMI } \\
\hline \multicolumn{12}{|l|}{$<23$} \\
\hline Model 1 & 1 & 0.85 & 0.48 & 1.51 & 1.04 & 0.62 & 1.74 & 0.97 & 0.54 & 1.74 & 0.92 \\
\hline Model 2 & 1 & 0.87 & 0.49 & 1.53 & 1.05 & 0.62 & 1.77 & 0.89 & 0.49 & 1.62 & 0.82 \\
\hline Model 3 & 1 & 0.91 & 0.51 & 1.60 & 1.03 & 0.61 & 1.76 & 0.92 & 0.51 & 1.68 & 0.89 \\
\hline Model 4 & 1 & 0.86 & 0.48 & 1.55 & 0.98 & 0.56 & 1.70 & 0.91 & 0.49 & 1.71 & 0.88 \\
\hline \multicolumn{12}{|l|}{$\geq 23$} \\
\hline Model 1 & 1 & 0.84 & 0.59 & 1.20 & 0.59 & 0.40 & 0.85 & 0.68 & 0.47 & 1.00 & 0.043 \\
\hline Model 2 & 1 & 0.84 & 0.59 & 1.19 & 0.58 & 0.40 & 0.84 & 0.67 & 0.45 & 0.99 & 0.035 \\
\hline Model 3 & 1 & 0.83 & 0.59 & 1.19 & 0.55 & 0.37 & 0.80 & 0.62 & 0.42 & 0.92 & 0.015 \\
\hline Model 4 & 1 & 0.79 & 0.55 & 1.14 & 0.53 & 0.36 & 0.78 & 0.61 & 0.41 & 0.92 & 0.018 \\
\hline
\end{tabular}

Model 1: adjusted for age groups (35-44, 45-54, 55-64, 65-74, $\geq 75$ years old) and gender.

Model 2: Model 1 plus body mass index $\left(<18,18-20.9,21-22.9,23-24.9\right.$, or $\left.\geq 25 \mathrm{~kg} / \mathrm{m}^{2}\right)$.

Model 3: Model 2 plus metabolic syndrome.

Model 4: Model 3 plus smoking, current alcohol drinking (regular/no), marital status (single, married and living with spouse, or divorced and separated), education level (less than 9 years, at least 9 years), occupation (no work, labor, official or business), hypertension status, HDL-C, triglycerides, glucose levels and family history of diabetes.

to a low risk pattern, including physical activity, diet, body mass index, smoking and alcohol intake [2]. In addition, physical inactivity has been associated with metabolic syndrome prevalence among the general adult population in the US [20]. Low levels of leisure-time physical activity resulted in the development of metabolic syndrome among 612 middleaged men after 4-years of follow-up in the Kuopio Ischemic Heart Disease Risk Factor Study [19], and the metabolic derangement risk was worse among participants with poor cardiorespiratory fitness [40]. In fact, a recent study on Chinese women has provided important data on the physical activity effect on diabetes risk [7]. In that study, leisure-time physical activity intensity provided a marginally protective effect on diabetes risk among 70,658 Chinese women in Shanghai who were followed for 4.6 years. In addition, daily living physical activity was inversely associated with diabetes risk in unemployed women.
The mechanism of the protective effects of physical activity on diabetes risk is explained by body weight control, improvement of biomarker profiles and reduction of metabolic syndrome risk. A cross-sectional analysis of 27,158 healthy women in the Women's health study showed that lower levels of physical activity were associated with adverse levels of nearly all lipid and inflammatory biomarkers [41]. Exercise and dietary restrictions improved weight control in a 2-year follow-up program [42], and the effect of exercise on weight loss was dose-respondent [43]. Furthermore, a recent randomized controlled trial demonstrated that physical activity effectively increased cardiorespiratory fitness among high risk women who were sedentary, overweight and postmenopausal: exercise training had a dose-response increase in physical fitness [44]. Even in an elderly population, regular exercise provided a significant reduction of metabolic risk factors and coronary heart disease [45]. Even without dietary restrictions, 


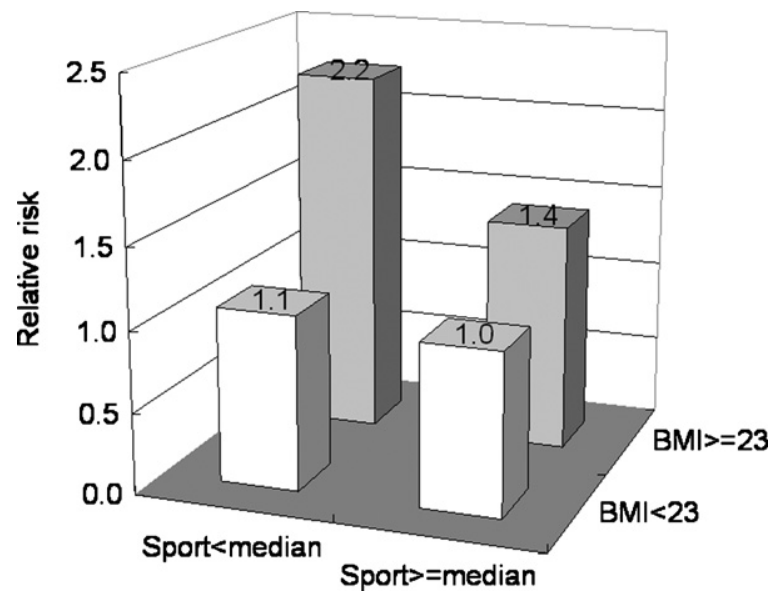

Fig. 1 - Relative risks of diabetes during 9 years of follow-up according to sports activity levels and body mass index, adjusted for age, gender, metabolic syndrome, smoking, current alcohol drinking, marital status, education level, occupation, hypertension status, HDL-C, triglycerides, glucose levels and family history of diabetes.

daily walking can improve biomarkers and lipid profiles among moderately obese cardiac patients [15]. Among a small study on obese Japanese males, daily walking alone could reduce visceral adipose tissue areas and improve insulin sensitivity [16]. A randomized controlled trial has shown that weight loss induced by increased daily physical activity without caloric restriction substantially reduced abdominal obesity and insulin resistance in men [46]. In another randomized control trial in 54 young women with abdominal obesity, a 14-week exercise intervention lead to a substantial reduction in total fat and insulin resistance [47]. Physical activity intensity and cardiorespiratory fitness have been inversely associated with metabolic syndrome frequency $[4,48]$. The cross-sectional Whitehall II study of middle-aged civil servants also showed that self-reported vigorous physical activity reduced the risk of metabolic syndrome by $48 \%$ [49].

Obesity and physical inactivity together account for nearly $60 \%$ of deaths from cardiovascular disease among women [36]. The magnitude of diabetes risk contributed by obesity is much greater than that imparted by lack of physical activity $[50,51]$. Our data did not show a significant association between various physical activities and body mass index, nor was an obesity modifying effect observed. One explanation is due to our relatively elderly population whose habitual activities in cross-sectional data were not reflected in the questionnaire. However, our combined results support an additive effect of obesity and physical inactivity on further diabetes risk, which is compatible with coronary heart disease risk in women [52]. In addition, our subgroup analysis showed that high sports activity was inversely associated with type 2 diabetes risk among participants with high body mass index. This finding implies that exercise may be beneficial for the obese population. Community-based randomized controlled trials have shown physical activity significantly prevents the risk of type 2 diabetes [53-55], and the effects are more prominent among the obese group.
Furthermore, our data shows the cutpoint of high body mass index to be 23, which is much lower than in Caucasians. Because the percentage of body fat is higher in Asians than in Caucasians with the same body mass index [56], Asians tended to develop type 2 diabetes and cardiovascular disease at body mass index levels lower than current World Health Organization cutoff points for the overweight $\left(25 \mathrm{~kg} / \mathrm{m}^{2}\right)$ [57]. Our data could be used as justification to recommend lower cutoff values for overweight and obesity in Asian populations, especially in physical activity promotion.

Several possibilities explain our findings about sports activity, not work or leisure-time activity, being associated with the risk of type 2 diabetes. First, sports activity included aerobic and anaerobic exercise which increases muscle training and energy expenditure [18], which facilitates body weight control. Even a single bout of high-intensity exercise can enhance glucose utilization in sedentary men [58]. Second, the evidence on association of different physical activity patterns is mixed. Among Japanese men, work physical activity, brisk walking and riding on vehicles were protective factors for developing diabetes [6]. However, work physical activity was not associated with type 2 diabetes risk among Chinese women workers [7]. Furthermore, leisure-time physical activity was marginally significant for diabetes risk in this study $(P$ trend $=0.12$ ). Third, potential measurement errors by a single subjective questionnaire reduced the power of the association so that only a marginally significant relationship was found in sports activity. Further studies on objective methods and different dimensions of physical activity, including type, duration, frequency and intensity, are warranted.

To the best of our knowledge, this is the first prospective investigation on physical activity and risk of diabetes among both genders in an ethnic Chinese population. Because of the prospective cohort design, the baseline measurements of all cohort members were unlikely to be affected by storage and laboratory issues that might be raised in some cross-sectional studies. Also, the use of a community-based population reduced the possibility of selection bias. We also included important socioeconomic and lifestyle factors in the models to control for potential confounding factors.

Our study has several potential limitations. First, we did not provide the data about maximal workload achieved (maximal metabolic equivalent) in the study participants so that potential misclassification might exist. Due to nondifferential misclassification, the actual magnitude of association between physical activity and diabetes risk might be more significant. Second, we did not measure cardiorespiratory fitness, which is defined as total time in a maximal treadmill exercise test. Previous studies have demonstrated that cardiorespiratory fitness predicts further metabolic syndrome risk, diabetes and cardiovascular disease $[19,40,59,60]$. Third, we did not collect detailed dietary intake questionnaire data in our baseline cohort. The potential additional effects of dietary behavior were therefore not evaluated in this study. Finally, transportation-related physical activity in an important component, but not included in the Baecke questionnaire.

This prospective study suggests an inverse association between sports activity and incidence of type 2 diabetes in a Chinese population. This association was persistent after adjustment for metabolic syndrome, and obesity increased 
the risk additively. Furthermore, because of only minimal correlation coefficients between physical activities and metabolic syndrome traits $(<0.1)$, we recommend that physical activities be measured for comprehensive evaluation of type 2 diabetes risk in Asian populations.

\section{Acknowledgements}

We thank the participants in the Chin-Shan community and the cardiologists at National Taiwan University Hospital for their assistance in this study. We make the disclosure of funding received for this work partly by grants from the National Science Council in Taiwan (NSC 96-2314-B-002-155and NSC 97-2314-B-002 -130-MY3).

\section{Conflict of interest}

The authors declare that they have no conflict of interest.

\section{R E F E R E N C E S}

[1] P. Zimmet, K.G. Alberti, J. Shaw, Global and societal implications of the diabetes epidemic, Nature 414 (2001) 782-787.

[2] F.B. Hu, J.E. Manson, M.J. Stampfer, G. Colditz, S. Liu, C.G. Solomon, et al., Diet, lifestyle, and the risk of type 2 diabetes mellitus in women, N. Engl. J. Med. 345 (2001) 790-797.

[3] G. Hu, Q. Qiao, K. Silventoinen, J.G. Eriksson, P. Jousilahti, J. Lindstrom, et al., Occupational, commuting, and leisuretime physical activity in relation to risk for type 2 diabetes in middle-aged Finnish men and women, Diabetologia 46 (2003) 322-329.

[4] S. Bertrais, J.P. Beyeme-Ondoua, S. Czernichow, P. Galan, S. Hercberg, J.M. Oppert, Sedentary behaviors, physical activity, and metabolic syndrome in middle-aged French subjects, Obes. Res. 13 (2005) 936-944.

[5] P.W. Sullivan, E.H. Morrato, V. Ghushchyan, H.R. Wyatt, J.O. Hill, Obesity, inactivity, and the prevalence of diabetes and diabetes-related cardiovascular comorbidities in the U.S., 2000-2002, Diabetes Care 28 (2005) 1599-1603.

[6] N. Nakanishi, T. Takatorige, K. Suzuki, Daily life activity and risk of developing impaired fasting glucose or type 2 diabetes in middle-aged Japanese men, Diabetologia 47 (2004) 1768-1775.

[7] R. Villegas, X.O. Shu, H. Li, G. Yang, C.E. Matthews, M. Leitzmann, et al., Physical activity and the incidence of type 2 diabetes in the Shanghai women's health study, Int. J. Epidemiol. 35 (2006) 1553-1562.

[8] L.B. Andersen, P. Schnohr, M. Schroll, H.O. Hein, All-cause mortality associated with physical activity during leisure time, work, sports, and cycling to work, Arch. Intern. Med. 160 (2000) 1621-1628.

[9] J. Fang, J. Wylie-Rosett, H.W. Cohen, R.C. Kaplan, M.H. Alderman, Exercise, body mass index, caloric intake, and cardiovascular mortality, Am. J. Prev. Med. 25 (2003) 283-289.

[10] N. Haapanen-Niemi, S. Miilunpalo, M. Pasanen, I. Vuori, P. Oja, J. Malmberg, Body mass index, physical inactivity and low level of physical fitness as determinants of all-cause and cardiovascular disease mortality-16 y follow-up of middle-aged and elderly men and women, Int. J. Obes. Relat. Metab. Disord. 24 (2000) 1465-1474.

[11] J.E. Manson, F.B. Hu, J.W. Rich-Edwards, G.A. Colditz, M.J. Stampfer, W.C. Willett, et al., A prospective study of walking as compared with vigorous exercise in the prevention of coronary heart disease in women, N. Engl. J. Med. 341 (1999) 650-658.

[12] J.E. Manson, P. Greenland, A.Z. LaCroix, M.L. Stefanick, C.P. Mouton, A. Oberman, et al., Walking compared with vigorous exercise for the prevention of cardiovascular events in women, N. Engl. J. Med. 347 (2002) 716-725.

[13] P.K. Myint, R.N. Luben, N.J. Wareham, A.A. Welch, S.A. Bingham, N.E. Day, et al., Combined work and leisure physical activity and risk of stroke in men and women in the European prospective investigation into cancer-norfolk prospective population study, Neuroepidemiology 27 (2006) 122-129.

[14] F.B. Hu, M.J. Stampfer, G.A. Colditz, A. Ascherio, K.M. Rexrode, W.C. Willett, et al., Physical activity and risk of stroke in women, JAMA 283 (2000) 2961-2967.

[15] D.J. Mertens, T. Kavanagh, R.B. Campbell, R.J. Shephard, Exercise without dietary restriction as a means to longterm fat loss in the obese cardiac patient, J. Sports Med. Phys. Fitness 38 (1998) 310-316.

[16] N. Miyatake, H. Nishikawa, A. Morishita, M. Kunitomi, J. Wada, H. Suzuki, et al., Daily walking reduces visceral adipose tissue areas and improves insulin resistance in Japanese obese subjects, Diabetes Res. Clin. Pract. 58 (2002) 101-107.

[17] F.B. Hu, Phyical activity, sedentary behaviors, and obesity, in: F.B. Hu (Ed.), Obesity Epidemiology, Oxford University Press, Oxford, 2008, pp. 301-319.

[18] C.J. Caspersen, K.E. Powell, G.M. Christenson, Physical activity, exercise, and physical fitness: definitions and distinctions for health-related research, Public Health Rep. 100 (1985) 126-131.

[19] D.E. Laaksonen, H.M. Lakka, J.T. Salonen, L.K. Niskanen, R. Rauramaa, T.A. Lakka, Low levels of leisure-time physical activity and cardiorespiratory fitness predict development of the metabolic syndrome, Diabetes Care 25 (2002) 16121618.

[20] E.S. Ford, H.W. Kohl 3rd, A.H. Mokdad, U.A. Ajani, Sedentary behavior, physical activity, and the metabolic syndrome among U.S. adults, Obes. Res. 13 (2005) 608-614.

[21] K.L. Chien, W.J. Chen, H.C. Hsu, T.C. Su, M.F. Chen, Y.T. Lee, Major gene effects on apolipoprotein B concentrations in families of adolescents-results from a community-based study in Taiwan, Clin. Chim. Acta 365 (2006) 194-199.

[22] K.L. Chien, F.C. Sung, H.C. Hsu, T.C. Su, R.S. Lin, Y.T. Lee, Apolipoprotein $\mathrm{A} 1$ and $\mathrm{B}$, and stroke events in a community-based cohort in Taiwan: report of Chin-Shan community cardiovascular study, Stroke 33 (2002) 39-44.

[23] Y.T. Lee, R.S. Lin, F.C. Sung, C.Y. Yang, K.L. Chien, W.J. Chen, et al., Chin-Shan community cardiovascular cohort in Taiwan: baseline data and five-year follow-up morbidity and mortality, J. Clin. Epidemiol. 53 (2000) 836-846.

[24] Y.T. Lee, F.C. Sung, R.S. Lin, H.C. Hsu, K.L. Chien, C.Y. Yang, et al., Peripheral blood cells among community residents living near nuclear power plants, Sci. Total Environ. 280 (2001) 165-172.

[25] K.L. Chien, M.F. Chen, H.C. Hsu, W.T. Chang, T.C. Su, Y.T. Lee, et al., Plasma uric acid and the risk of type 2 diabetes in a Chinese community, Clin. Chem. 54 (2008) 310-316.

[26] J.A. Baecke, J. Burema, J.E. Frijters, A short questionnaire for the measurement of habitual physical activity in epidemiological studies, Am. J. Clin. Nutr. 36 (1982) 936-942.

[27] M.A. Pols, P.H. Peeters, H.B. Bueno-De-Mesquita, M.C. Ocke, C.A. Wentink, H.C. Kemper, et al., Validity and repeatability 
of a modified Baecke questionnaire on physical activity, Int. J. Epidemiol. 24 (1995) 381-388.

[28] W.H. Pan, Y.T. Hung, N.S. Shaw, W. Lin, S.D. Lee, C.F. Chiu, et al., Elderly nutrition and health survey in Taiwan (19992000): research design, methodology and content, Asia Pac. J. Clin. Nutr. 14 (2005) 203-210.

[29] K.L. Chien, Y.T. Lee, F.C. Sung, H.C. Hsu, T.C. Su, R.S. Lin, Hyperinsulinemia and related atherosclerotic risk factors in the population at cardiovascular risk: a communitybased study, Clin. Chem. 45 (1999) 838-846.

[30] K.L. Chien, H.C. Hsu, F.C. Sung, T.C. Su, M.F. Chen, Y.T. Lee, Hyperuricemia as a risk factor on cardiovascular events in Taiwan: the Chin-Shan community cardiovascular cohort study, Atherosclerosis 183 (2005) 147-155.

[31] H. Wieland, D. Seidel, A simple specific method for precipitation of low density lipoproteins, J. Lipid Res. 24 (1983) 904-909.

[32] F.B. Hu, M.J. Stampfer, C. Solomon, S. Liu, G.A. Colditz, F.E. Speizer, et al., Physical activity and risk for cardiovascular events in diabetic women, Ann. Intern. Med. 134 (2001) 96105.

[33] C.E. Tan, S. Ma, D. Wai, S.K. Chew, E.S. Tai, Can we apply the national cholesterol education program adult treatment panel definition of the metabolic syndrome to Asians? Diabetes Care 27 (2004) 1182-1186.

[34] D.W. Hosmer Jr., S. Lemeshow, The multiple logistic regression model, in: Applied Logistic Regression, John Wiley \& Sons, New York, 1989, pp. 25-37.

[35] J. Hsia, L. Wu, C. Allen, A. Oberman, W.E. Lawson, J. Torrens, et al., Physical activity and diabetes risk in postmenopausal women, Am. J. Prev. Med. 28 (2005) 19-25.

[36] G. Hu, J. Lindstrom, T.T. Valle, J.G. Eriksson, P. Jousilahti, K. Silventoinen, et al., Physical activity, body mass index, and risk of type 2 diabetes in patients with normal or impaired glucose regulation, Arch. Intern. Med. 164 (2004) 892-896.

[37] G. Hu, J. Tuomilehto, K. Silventoinen, N. Barengo, P. Jousilahti, Joint effects of physical activity, body mass index, waist circumference and waist-to-hip ratio with the risk of cardiovascular disease among middle-aged Finnish men and women, Eur. Heart J. 25 (2004) 2212-2219.

[38] P.T. Katzmarzyk, C.L. Craig, L. Gauvin, Adiposity, physical fitness and incident diabetes: the physical activity longitudinal study, Diabetologia 50 (2007) 538-544.

[39] F.B. Hu, T.Y. Li, G.A. Colditz, W.C. Willett, J.E. Manson, Television watching and other sedentary behaviors in relation to risk of obesity and type 2 diabetes mellitus in women, JAMA 289 (2003) 1785-1791.

[40] T.A. Lakka, D.E. Laaksonen, H.M. Lakka, N. Mannikko, L.K. Niskanen, R. Rauramaa, et al., Sedentary lifestyle, poor cardiorespiratory fitness, and the metabolic syndrome, Med. Sci. Sports Exerc. 35 (2003) 1279-1286.

[41] S. Mora, I.M. Lee, J.E. Buring, P.M. Ridker, Association of physical activity and body mass index with novel and traditional cardiovascular biomarkers in women, JAMA 295 (2006) 1412-1419.

[42] C.L. Dunn, P.J. Hannan, R.W. Jeffery, N.E. Sherwood, N.P. Pronk, R. Boyle, The comparative and cumulative effects of a dietary restriction and exercise on weight loss, Int. J. Obes. (Lond.) 30 (2006) 112-121.

[43] R. Ross, I. Janssen, Physical activity, total and regional obesity: dose-response considerations, Med. Sci. Sports Exerc. 33 (2001) S521-S527, discussion S528-S529.

[44] T.S. Church, C.P. Earnest, J.S. Skinner, S.N. Blair, Effects of different doses of physical activity on cardiorespiratory fitness among sedentary, overweight or obese postmenopausal women with elevated blood pressure: a randomized controlled trial, JAMA 297 (2007) 2081-2091.
[45] R.J. Petrella, C.N. Lattanzio, A. Demeray, V. Varallo, R. Blore, Can adoption of regular exercise later in life prevent metabolic risk for cardiovascular disease? Diabetes Care 28 (2005) 694-701.

[46] R. Ross, D. Dagnone, P.J. Jones, H. Smith, A. Paddags, R. Hudson, et al., Reduction in obesity and related comorbid conditions after diet-induced weight loss or exerciseinduced weight loss in men. A randomized, controlled trial, Ann. Intern. Med. 133 (2000) 92-103.

[47] R. Ross, I. Janssen, J. Dawson, A.M. Kungl, J.L. Kuk, S.L. Wong, et al., Exercise-induced reduction in obesity and insulin resistance in women: a randomized controlled trial, Obes. Res. 12 (2004) 789-798.

[48] S. Carroll, C.B. Cooke, R.J. Butterly, Metabolic clustering, physical activity and fitness in nonsmoking, middle-aged men, Med. Sci. Sports Exerc. 32 (2000) 2079-2086.

[49] K.L. Rennie, N. McCarthy, S. Yazdgerdi, M. Marmot, E. Brunner, Association of the metabolic syndrome with both vigorous and moderate physical activity, Int. J. Epidemiol. 32 (2003) 600-606.

[50] J.S. Rana, T.Y. Li, J.E. Manson, F.B. Hu, Adiposity compared with physical inactivity and risk of type 2 diabetes in women, Diabetes Care 30 (2007) 53-58.

[51] A.R. Weinstein, H.D. Sesso, I.M. Lee, N.R. Cook, J.E. Manson, J.E. Buring, et al., Relationship of physical activity vs body mass index with type 2 diabetes in women, JAMA 292 (2004) 1188-1194.

[52] T.Y. Li, J.S. Rana, J.E. Manson, W.C. Willett, M.J. Stampfer, G.A. Colditz, et al., Obesity as compared with physical activity in predicting risk of coronary heart disease in women, Circulation 113 (2006) 499-506.

[53] G. Davey Smith, Y. Bracha, K.H. Svendsen, J.D. Neaton, S.M. Haffner, L.H. Kuller, Incidence of type 2 diabetes in the randomized multiple risk factor intervention trial, Ann. Intern. Med. 142 (2005) 313-322.

[54] A.K. Jenum, S.A. Anderssen, K.I. Birkeland, I. Holme, S. Graff-Iversen, C. Lorentzen, et al., Promoting physical activity in a low-income multiethnic district: effects of a community intervention study to reduce risk factors for type 2 diabetes and cardiovascular disease: a community intervention reducing inactivity, Diabetes Care 29 (2006) 1605-1612.

[55] I. Thune, I. Njolstad, M.L. Lochen, O.H. Forde, Physical activity improves the metabolic risk profiles in men and women: the Tromso study, Arch. Intern. Med. 158 (1998) 1633-1640.

[56] P. Deurenberg, M. Deurenberg-Yap, S. Guricci, Asians are different from Caucasians and from each other in their body mass index/body fat per cent relationship, Obes. Rev. 3 (2002) 141-146.

[57] Appropriate body-mass index for Asian populations and its implications for policy and intervention strategies, Lancet 363 (2004) 157-163.

[58] Y. Hayashi, S. Nagasaka, N. Takahashi, I. Kusaka, S. Ishibashi, S. Numao, et al., A single bout of exercise at higher intensity enhances glucose effectiveness in sedentary men, J. Clin. Endocrinol. Metab. 90 (2005) 40354040.

[59] M.J. LaMonte, C.E. Barlow, R. Jurca, J.B. Kampert, T.S. Church, S.N. Blair, Cardiorespiratory fitness is inversely associated with the incidence of metabolic syndrome: a prospective study of men and women, Circulation 112 (2005) 505-512.

[60] A.S. Leon, D. Casal, D. Jacobs Jr., Effects of $2000 \mathrm{kcal}$ per week of walking and stair climbing on physical fitness and risk factors for coronary heart disease, J. Cardiopulm. Rehabil. 16 (1996) 183-192. 